\title{
Application of Partitioned Iterative Coupling Approach to Multi-physics
}

\author{
Tomohide Takeyama, Shinya Tachibana, Tomoki Kitanoi, Atsushi lizuka \\ Kobe University, 1-1 Rokkodai Nada, Kobe, Hyogo 657-8501, Japan \\ E-mail:takeyama@people.kobe-u.ac.jp
}

Received: 4 September 2018; Accepted: 1 October 2018; Available online: 15 January 2019

\begin{abstract}
The engineering needs which have to consider the multi-physics are increasing. When the multi-phase problem is treated by monolithic coupling approach, there are some difficulties such as the increase in the number of degree of freedom, the complication of the program code and so on. In order to overcome these shortcomings in considering the new additional phase, we applied the partitioned iterative coupling approach to the HydroMechanical coupled problem in this research. In the partitioned iterative coupling approach, the behavior of single phases can be individually calculated and the interaction between the phases has been considered at an appropriate timing by the middleware. The middleware plays a role to regulate the single-phase analysis. In this research, we carried out the numerical simulation of one-dimensional consolidation by both the partitioned iterative coupling scheme and the monolithic coupling scheme, and then we confirmed that the numerical results are the same. Through the research, it figured out the efficient iterative scheme.
\end{abstract}

Keywords: Hydro-Mechanical coupling; Partitioned method; Multi-physics.

\section{Introduction}

When we analyze the behavior of saturated soil, it is necessary to consider the interaction between the deformation of the soil particle and the change in pore water pressure. Therefore, we have to solve simultaneously the governing equation such as the equilibrium equation and continuity equation.

In recent years, the structures and the phenomena to be analyzed has been diversified, and in some cases, pore air, heat, and chemistry also have to be coupled. The field dealing with such problems is called Multi-physics.

When we solve a problem with a large number of coupled phases, the degree of freedom of the simultaneous equations to be solved increases as the number of coupled phases increases. In addition, the program becomes complicated, it becomes difficult to maintain and improve, which may cause a bug. The method solved simultaneously the governing equations describing the coupled problem is called the monolithic method. On the other hand, the method which the governing equations concerning each phase is solved separately, and the convergence calculation is conducted is called the partitioned method (Jahromi, 2007 [1]). If the deformation of soil, pore water pressure, pore air pressure, heat, chemical reaction etc. can be solved separately by the partitioned method, we can connect existing software and use it effectively. As a result, we can overcome the above-mentioned shortcomings of a large number of coupled phase problem.

The objective of this research is to solve the soil-water coupled problem by the partitioned method. In the future, the partitioned method will be applied in the field of Multi-Physics other than soil-water. The applicability of the partitioned method to the one-dimensional consolidation problem is investigated in this research. Then, we compared the solution by the partitioned method and the monolithic method.

\section{Soil-water coupled problem and partitioned method}

The governing equations to describe the soil-water coupled problem are as follows. In the following description, compression is defined to be positive.

$$
\begin{aligned}
& \nabla \cdot \boldsymbol{\sigma}-\rho \boldsymbol{b}=0, \boldsymbol{\sigma}=\boldsymbol{\sigma}^{\prime}+p_{w} \mathbf{1}, \dot{\boldsymbol{\sigma}}^{\prime}=\boldsymbol{C}: \dot{\boldsymbol{\varepsilon}}, \boldsymbol{\varepsilon}=-(\boldsymbol{u} \otimes \nabla+\nabla \otimes \boldsymbol{u}) / 2 \\
& \boldsymbol{w}=-\boldsymbol{k} \cdot \nabla h, \dot{\varepsilon}_{v}-\nabla \cdot \boldsymbol{w}=0
\end{aligned}
$$

These equations are the equilibrium equation, the principle of effective stress, the constitutive equation, the strain-displacement relation, Darcy's law and the continuity equation respectively. $\rho$ is density, $\boldsymbol{u}$ is displacement vector, $\boldsymbol{\sigma}$ is stress tensor, $\boldsymbol{b}$ is body force vector, $\boldsymbol{\sigma}^{\prime}$ is effective stress tensor, $p_{w}$ is pore water pressure, $\boldsymbol{l}$ is the 
Kronecker delta, $C$ is constitutive tensor, $\boldsymbol{\varepsilon}$ is strain tensor, $\boldsymbol{w}$ is Darcy's velocity, $\boldsymbol{k}$ is hydraulic conductivity tensor, $h$ is total water head, $\varepsilon_{v}$ is volumetric strain. The boundary conditions are

$$
\begin{aligned}
& \widehat{\boldsymbol{u}}=\boldsymbol{u} \text { on } S_{u}, \hat{\boldsymbol{t}}=-\boldsymbol{\sigma}^{T} \cdot \boldsymbol{n} \text { on } S_{\sigma} \\
& \hat{h}=h \text { on } S_{h}, \hat{q}=\boldsymbol{w} \cdot \boldsymbol{n} \text { on } S_{q}
\end{aligned}
$$

where $\boldsymbol{t}$ is traction vector, $\boldsymbol{n}$ is a normal vector to the boundary surface, $q$ is a quantity of flowing pore water, $S_{u}$ is displacement boundary, $S_{\sigma}$ is stress boundary, $S_{h}$ is head boundary, $S_{q}$ is flow boundary.

By multiplying the rate form of equilibrium equation by a test function $\delta \dot{\boldsymbol{u}}$ and volume integrating it, the weak form of the equation is obtained:

$$
\int_{V} \delta \dot{\boldsymbol{\varepsilon}}: \dot{\boldsymbol{\sigma}}^{\prime} d V+\int_{V} \delta \dot{\varepsilon}_{v} \dot{p}_{w} d V-\int_{S_{\sigma}} \delta \dot{\boldsymbol{u}} \cdot \dot{\hat{\boldsymbol{t}}} d S-\int_{V} \rho \delta \dot{\boldsymbol{u}} \cdot \dot{\boldsymbol{b}} d V=0
$$

The variables are spatially discretized as shown below.

$$
\begin{aligned}
& \{u\}=[N]\left\{u^{n e}\right\},\{\varepsilon\}=[B]\left\{u^{n e}\right\},\left\{\varepsilon_{v}\right\}=\left[B_{v}\right]\left\{u^{n e}\right\}, \\
& h=\left[N_{h}\right]\left\{h^{m e}\right\},\{\nabla h\}=\left[B_{h}\right]\left\{h^{m e}\right\},
\end{aligned}
$$

where $[N],[B],\left[B_{v}\right],\left[N_{h}\right]$ and $\left[B_{h}\right]$ are the shape function matrices, the superscript $n e$ and me indicate the variables at the discretized points. By substituting these equations to the weak form of equilibrium equation and continuity equation, the spatially discretized equations of an element are obtained:

$$
\begin{aligned}
& {\left[K^{e}\right]\left\{\dot{u}^{n e}\right\}+\left[K_{v}^{e}\right]^{T}\left\{\gamma_{w} \dot{h}^{m e}\right\}=\left\{\dot{F}^{e}\right\}} \\
& {\left[K_{v}^{e}\right]\left\{\dot{u}^{n e}\right\}-\left[K_{h}^{e}\right]^{T}\left\{\gamma_{w} h^{m e}\right\}=\left\{\dot{Q}^{e}\right\}}
\end{aligned}
$$

where matrices are expressed as follows:

$$
\begin{aligned}
& {\left[K^{e}\right]=\int_{V^{e}}[B]^{T}[D][B] d V,\left[K_{v}^{e}\right]=\int_{V^{e}}\left[N_{h}\right]^{T}\left[B_{v}\right] d V,\left[K_{h}^{e}\right]=\int_{V}\left[B_{h}\right]^{T} \frac{[k]}{\gamma_{w}}\left[B_{h}\right] d V,} \\
& \left\{\dot{F}^{e}\right\}=\int_{S_{\sigma}^{e}}[N]^{T}\{\dot{\hat{t}}\} d S+\int_{V} \rho[N]^{T}\{\dot{b}\} d V,\left\{\dot{Q}^{e}\right\}=\int_{S_{q}^{e}}\left[N_{h}\right]^{T}\{\hat{q}\} d S
\end{aligned}
$$

The time derivative of displacement, the total water head and the time derivative of the total water head are temporally discretized as

$$
\dot{u}=\Delta u / \Delta t, h=(1-\theta) h_{t}+\theta h_{t+\Delta t}, \dot{h}=\left(h_{t+\Delta t}-h_{t}\right) / \Delta t
$$

where $\theta$ is constant within a range from 0 to 1 . By substituting these equations to spatially discretized equations and summing up all elements, the spatially and temporally discretized weak forms of the equilibrium equation and the continuity equation (the global stiffness equation) are obtained:

$$
\left[\begin{array}{cc}
{[K]} & {\left[K_{v}\right]^{T}} \\
{\left[K_{v}\right]} & -\theta \Delta t\left[K_{h}\right]
\end{array}\right]\left\{\begin{array}{c}
\left\{\Delta u^{n}\right\} \\
\left\{\gamma_{w} h_{t+\Delta t}^{m}\right\}
\end{array}\right\}=\left\{\begin{array}{c}
\Delta t\{\dot{F}\}+\left[K_{v}\right]^{T}\left\{\gamma_{w} h_{t}^{m}\right\} \\
\Delta t\{Q\}+(1-\theta) \Delta t\left[K_{h}\right]\left\{\gamma_{w} h_{t}^{m}\right\}
\end{array}\right\}
$$

In order to separate the phase, the terms other than the diagonal terms are shifted to the right-hand side of the equation, we obtain

$$
\left[\begin{array}{cc}
{[K]} & 0 \\
0 & -\theta \Delta t\left[K_{h}\right]
\end{array}\right]\left\{\begin{array}{c}
\left\{\Delta u^{n}\right\} \\
\left\{\gamma_{w} h_{t+\Delta t}^{m}\right\}
\end{array}\right\}=\left\{\begin{array}{c}
\Delta t\{\dot{F}\}+\left[K_{v}\right]^{T}\left\{\gamma_{w} h_{t}^{m}\right\}-\left[K_{v}\right]^{T}\left\{\gamma_{w} h_{t+\Delta t}^{m}\right\} \\
\Delta t\{Q\}+(1-\theta) \Delta t\left[K_{h}\right]\left\{\gamma_{w} h_{t}^{m}\right\}-\left[K_{v}\right]\left\{\Delta u^{n}\right\}
\end{array}\right\}
$$

The upper equation describes the deformation problem and the shifted terms are given as the external force which is corresponding with the calculated excess pore water pressure from the lower equation. On the contrary, the lower equation describes the seepage flow problem and the shifted terms are given as the quantity of water flow which is corresponding to the calculated increment of displacement from the upper equation. These equations are solved separately until the solutions are converged. Fig. 1 shows a flowchart of the above mentioned partitioned 
method for soil-water coupled problem introduced the SOR method (Young, 1971 [2], Masatake, 2002 [3]). In Fig.1, the matrixes and vectors are replaced to simplify as

$$
\begin{aligned}
& {[K] \rightarrow\left[K_{u u}\right],\left[K_{v}\right]^{T} \rightarrow\left[K_{u h}\right],\left[K_{v}\right] \rightarrow\left[K_{h u}\right],-\theta \Delta t\left[K_{h}\right] \rightarrow\left[K_{h h}\right]} \\
& \Delta t\{\dot{F}\}+\left[K_{v}\right]^{T}\left\{\gamma_{w} h_{t}^{m}\right\} \rightarrow\{F\}, \Delta t\{Q\}+(1-\theta) \Delta t\left[K_{h}\right]\left\{\gamma_{w} h_{t}^{m}\right\} \rightarrow\{Q\}
\end{aligned}
$$

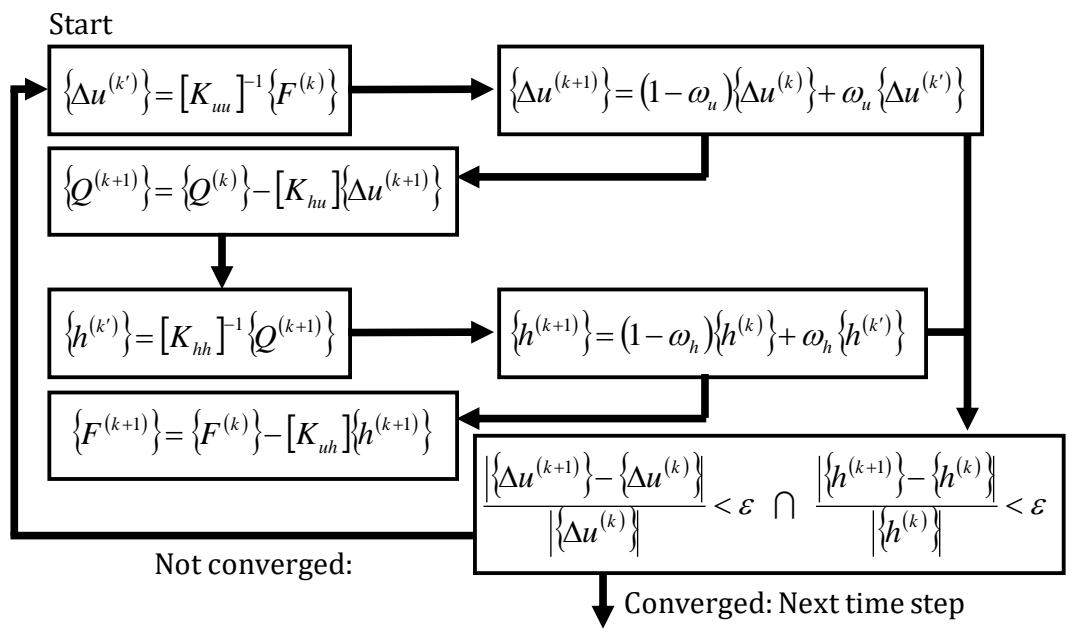

Fig. 1: Flowchart of the partitioned method for soil-water coupled problem

$\omega_{u}$ and $\omega_{h}$ are the acceleration parameter to be slow the convergence speed for the increment of displacement $\Delta u$ and the hydraulic head $h$ respectively, and they are applied in order to prevent from the divergence of calculation.

\section{Verification of partitioned method in one-dimensional consolidation}

In this section, the applicability of the partitioned method to soil-water coupled problem is verified by comparing the simulated results of one-dimensional consolidation obtained by the partitioned method and the monolithic method. In the simulation, one element is used. The upper side of the element is drained condition and the other sides are set as undrained conditions. The linear elastic constitutive model is applied in the simulation. The Lame's constant $\lambda$ and $\mu$ are set to be $1338.8 \mathrm{kN} / \mathrm{m}^{2}$ and $666.9 \mathrm{kN} / \mathrm{m}^{2}$ respectively. The coefficient of permeability $k$ is set to be $1.0 \times 10^{-4} \mathrm{~m} / \mathrm{s}$. The load of $98 \mathrm{kPa}$ is applied to the upper side of the element at the first time step which the increment of time $\Delta t=1.0 \times 10^{-3}$ (day). After the second time step, the displacement at the upper side of the element and the excess pore water pressure at the lower side of the element are calculated by giving the increment of time $\Delta t=0.0367$ (day) which is corresponded to the time factor $T_{v}=0.001$ and repeating the step 1000 times.

The convergence calculation is carried out at the first step in the consolidation problem which the elapsed time is 0.001 (day) by using the partitioned method. In this research, when the error rate which is defined as $\left|x^{(k+1)}-x^{(k)}\right| / \mid$ $x^{(k)} \mid \times 100$ becomes less than or equals to $1.0 \times 10^{-5}$, the solutions are judged to be converged.

By changing the acceleration parameter $\omega_{u}$ from $1.0 \times 10^{-6}$ to $1.0 \times 10^{-3}$ by each 0.1 and the acceleration parameter $\omega_{h}$ from 0.1 to 0.5 by each 0.05 , the convergence calculation was performed and the iteration number was obtained. Fig. 2 shows a contour diagram of the iteration number. The interval between contour lines is every 20 iterations, after 200 iterations, every 100 iterations. When $\omega_{u}=1.0 \times 10^{-3}$, the calculation diverged between 0.4 and 0.5 of $\omega_{h}$. When $\omega_{u}=1.0 \times 10^{-3}$, the calculation was not converged within 500 iterations. It is understandable results that the calculation is diverged by using a large acceleration parameter and conversely, the number of iteration increases by using a small acceleration parameter. In this case, the optimum acceleration parameter $\omega_{\text {uopt }}, \omega_{\text {hopt }}$ becomes $1.0 \times 10^{-4}, 0.45$ respectively.

Fig. 3 shows the contour diagram of the iteration number in case of $\Delta t=0.0356$ (day) for the second step. By changing the acceleration parameter $\omega_{u}$ from $1.0 \times 10^{-4}$ to $1.0 \times 10^{-1}$ by each 0.1 and the acceleration parameter $\omega_{h}$ from 0.1 to 0.6 by each 0.05 , the convergence calculation was performed. The trend in which the divergence occurs when a large acceleration parameter is used and the convergence becomes slower when the smaller acceleration parameter is used is similar to the first step. However, the appropriate acceleration parameter is different from the first step. In this case, the optimum acceleration parameter $\omega_{\text {uopt }}, \omega_{\text {hopt }}$ becomes $1.0 \times 10^{-3}, 0.55$ respectively. 


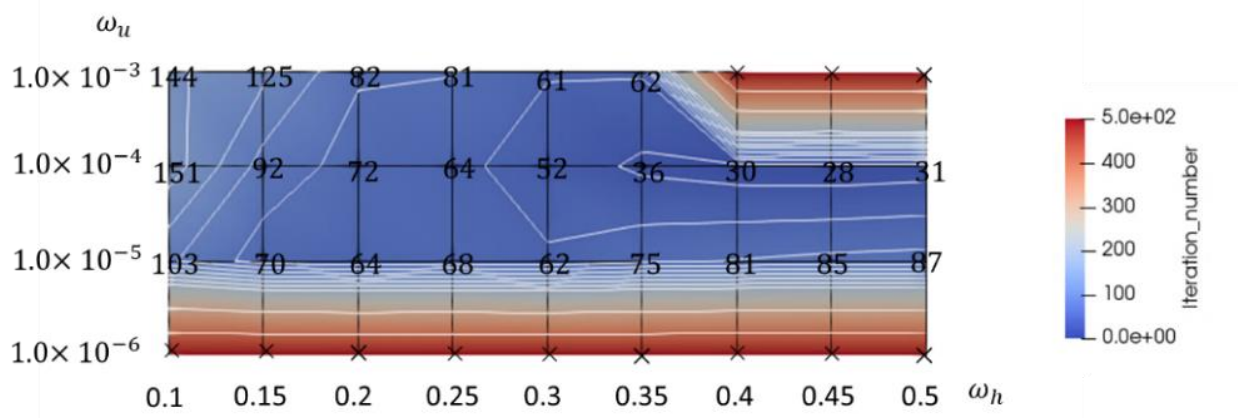

Fig. 2: The contour diagram of $\Delta t=0.001$ (day)

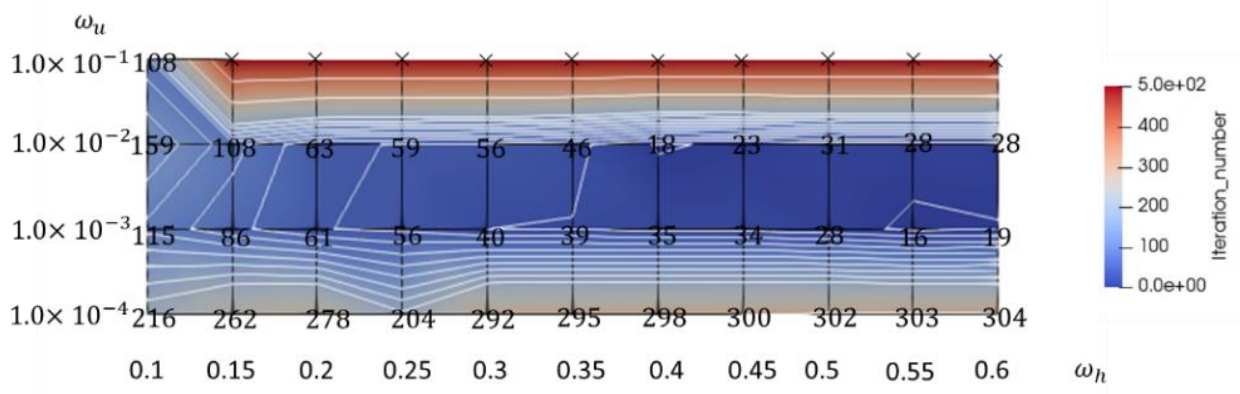

Fig. 3: The contour diagram of $\Delta t=0.0356$ (day)

This result suggests that the optimum value of the acceleration parameter has changed due to the change in the time increment between the first step and second step. Since $\left[K_{h h}\right]$ matrix contains the time increment, we investigated the influence of the order of $\left[\mathrm{K}_{\mathrm{hh}}\right]$ matrix in iteration number of the convergence calculation.

Figs. 4 to 6 show the contour diagram of the iteration number when the order of norm of $\left[\mathrm{K}_{\mathrm{hh}}\right]$ matrix is set to be $10^{-9}, 10^{-10}, 10^{-11}$ respectively. The contour diagram is shown in Fig. 5 is same as the result of the convergence calculation at the first step. When the order of norm of $\left[\mathrm{K}_{\mathrm{hh}}\right]$ matrix becomes 10 times larger or 10 times smaller, the contour lines move along the axis of the acceleration parameter for the displacement $\omega_{u}$. The appropriate acceleration parameter $\omega_{u}$ is $1.0 \times 10^{-5}, 1.0 \times 10^{-4}, 1.0 \times 10^{-3}$ in case of $\left\|\mathrm{K}_{\mathrm{hh}}\right\|=\mathrm{O}\left(10^{-9}\right), \mathrm{O}\left(10^{-10}\right), \mathrm{O}\left(10^{-11}\right)$ respectively. From these cases, the appropriate acceleration parameter is obviously related with the order of $\left[\mathrm{K}_{\mathrm{hh}}\right]$ matrix. These results suggest that the appropriate acceleration parameter might be possible to be estimated by $\left[\mathrm{K}_{\mathrm{hh}}\right]$ matrix.

A comparison between the results of one-dimensional consolidation obtained by the partitioned method and the results obtained by the monolithic method is shown in Fig. 7. In order to calculate the displacement and excess pore water pressure by the monolithic method, the finite element program which can solve the soil-water coupled problem named DACSAR-I (Iizuka et al., 1987 [4], Takeyama et al. 2015 [5]) is employed. In the partitioned method, the appropriate acceleration parameters $\omega_{u}$ and $\omega_{h}$ set to be $1.0 \times 10^{-4}, 0.45$ for the first step and $1.0 \times 10^{-3}$, 0.55 for the second step and after based on the contour diagram shown in Fig. 2 and Fig. 3. From Fig. 7, it is confirmed that the results by the partitioned method and the results by the monolithic method agree with each other. It is found that the disadvantage of the partitioned method that it takes a lot of time because there is processing of reading and writing the file in the iteration process.

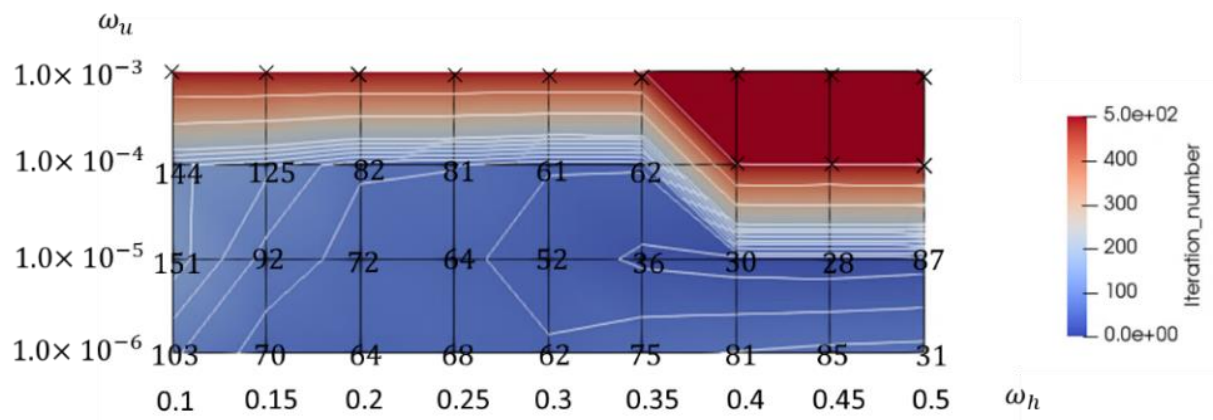

Fig. 4: The contour diagram of $\left\|\left[K_{h \boldsymbol{h}}\right]\right\|=\sigma\left(\mathbf{1 0}^{-\mathbf{9}}\right)$ 
$\omega_{u}$

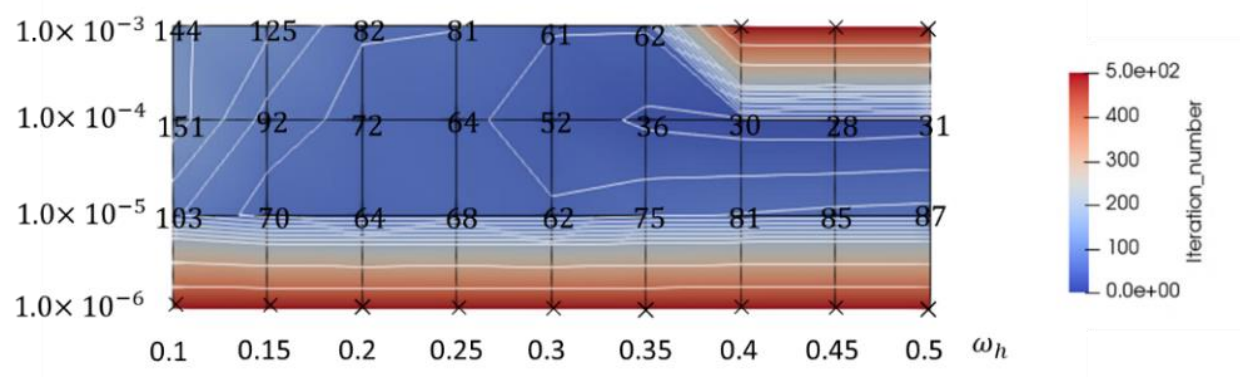

Fig. 5: The contour diagram of $\left\|\left[K_{h \boldsymbol{h}}\right]\right\|=\sigma\left(\mathbf{1 0}^{-\mathbf{1 0}}\right)$

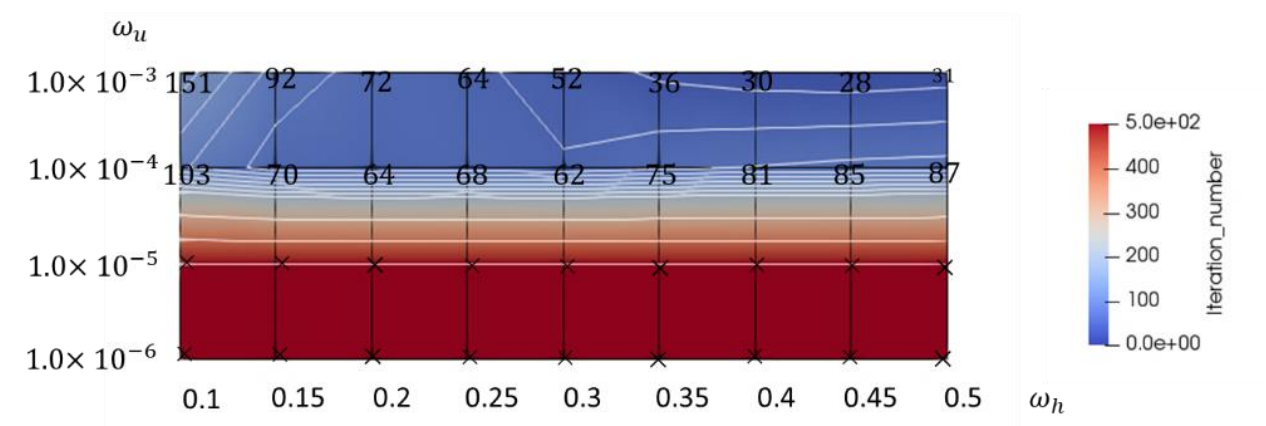

Fig. 6: The contour diagram of $\left\|\left[K_{h h}\right]\right\|=\sigma\left(\mathbf{1 0}^{-11}\right)$
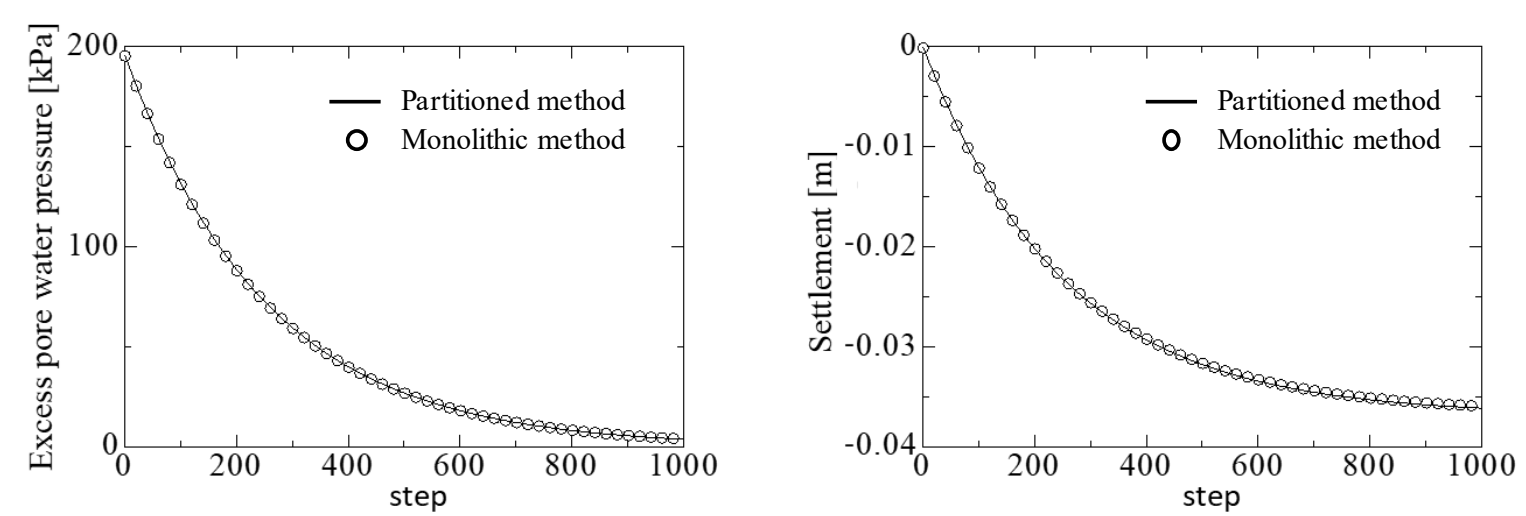

Fig. 7: Comparison between the partitioned method and the monolithic method

\section{Conclusions}

In order to solve the soil-water coupled problem separately, the partitioned method was applied. The coupled problem is divided into the deformation problem and the seepage flow problem and are solved separately until the solutions are converged. In order to verify the applicability of the partitioned method to soil-water coupled problem, the simulated results of one-dimensional consolidation obtained by the partitioned method and the results by the monolithic method are compared. The acceleration parameter is adopted in the iterative calculation. The appropriate acceleration parameter is obviously related with the order of the diagonal matrix in the global stiffness matrix. Therefore it is suggested that the appropriate acceleration parameters might be possible to be estimated by the diagonal matrix. It is confirmed that the results by the partitioned method and the results by the monolithic method agree with each other. However, it is found that the disadvantage of the partitioned method that it takes a lot of time because there is processing of reading and writing the file in the iterative calculation.

\section{References}


[1] Zolghadr Jahromi H. Partitioned analysis of nonlinear soil-structure iteration using iterative coupling. Interaction and Multiscale. 2007;1(1):33-51.

[2] Young DM. Iterative solution of large linear systems. New York: Academic Press; 1971.

[3] Masatake M. The numerical analysis. 2002.

[4] Iizuka A, Ohta H. A determination procedure of input parameters in elasto-viscoplastic finite element analysis. Soils and foundations. 1987; 27(3):71-87.

[5] Takeyama T, Tachibana S, Furukawa A. A finite element method to describe the cyclic behavior of saturated soil. International Journal of Material Science and Engineering. 2015;2(1): 20-25.

(C) 2019 by the author(s). This work is licensed under a Creative Commons Attribution 4.0 International License (http://creativecommons.org/licenses/by/4.0/). Authors retain copyright of their work, with first publication rights granted to Tech Reviews Ltd. 